\title{
Surgeons as advocates for opioid control
}

The views expressed in this editorial are those of the author and do not necessarily reflect the position of the Canadian Medical Association or its subsidiaries.

ecently, an increasing number of reports have been warning physicians about the dangers of prescribing opioids. This is particularly relevant to our surgical community, given the traditional reliance on this category of medications as postoperative pain relievers. Unfortunately, there is growing evidence in both the formal scientific and popular mainstream literature that the risks of narcotic prescriptions may in fact be doing substantially more harm to our patients than we had ever understood.

Although details outlining the origins of the Sackler brothers' (both psychiatrists) initial formulation of OxyContin have been locked away for decades in a shadowy, propaganda-laden box, more than 200000 Americans have died of OxyContin overdoses since 1996. In 2016 alone, 53000 Americans died from opioid overdoses, compared with 36000 and 35000 who died from motor vehicle crashes and gunshot wounds, respectively. For those of us who deal with injured patients on a daily basis, these numbers are comparatively staggering.

While American media dominate this discussion, there are recent data arguing that we may have an even larger opioid crisis in Canada. More specifically, within the first 6 months of 2018 alone, there were 2066 opioidrelated deaths in Canada (11.2 deaths per 100000 people). ${ }^{1}$ Across the country, Calgary consistently has the highest opioid-related death rate at 18.7 per 100000 population (687 deaths in 2017) as well as the highest emergency department opioid-related visit rate $(8 \%-$ $10 \%$ of all visits over the past 3 years). ${ }^{1,2}$ Other provinces are not exempt from this public health issue either, given that Ontario leads the country in both total opioid prescriptions (9 million prescriptions per year) and total opioid-related deaths (1250 in 2017). ${ }^{3}$ Furthermore, Canada is the largest per capita consumer of prescription opioids and second largest prescriber of opioids (35000 per year) in the developed world. ${ }^{4}$ One in 7 Canadians fills an opioid prescription each year. This is particularly problematic, considering that up to $75 \%$ of all heroin users are first introduced to narcotics through an initial physician- or surgeonrelated opioid prescription. ${ }^{5}$
The dramatic rise in opioid-related deaths (350\% increase between 2003 and 2017) mirrors a number of noncoincidental realities that have occurred over this time frame:

- Big Pharma, the American Pain Society, and the American Academy of Pain Medicine (among others) pressured governments and physicians alike to make pain "the fifth vital sign" (with a 10-point associated pain scale). By intensifying the more broad meaning of pain, internal Pharma documentation clearly aims at "attaching an emotional aspect to non-cancer pain" so that physicians feel the need to treat it more aggressively.

- A dizzying variety in opioid types and formulations became much more diverse and therefore challenging to manage.

- The increase in potency of newer narcotics has been significant (the oral morphine equivalent [OME] of hydromorhone [2 $\mathrm{mg}$ tablet] is 80 , whereas that of codeine [ $30 \mathrm{mg}$ tablet] is 45 ).

- Addicts have figured out how to manipulate opioid pills to be consumed in a wide variety of different delivery methods (e.g., ingestion, smoking, inhalation).

- Addicts who could not afford the street cost of many prescribed opioids switched to using more traditional narcotics, such as heroin.

- A strategic and persistent campaign to misinform physicians regarding the abuse potential of many of these opioids (which was successfully litigated by the United States government in 2007) was extremely potent.

Although as surgeons we often believe that our prescription patterns (and therefore patient usage) involve only short-term effects, it is clear that orthopedic surgeons, neurosurgeons and gastrointestinal surgeons consistently make up the top 3 physician prescriber groups. ${ }^{6}$ The US Centers for Disease Control and Prevention (CDC) stated that opioid use for longer than 5 days is a significant risk factor for subsequent narcotic misuse. ${ }^{6}$ Furthermore, more than $10 \%$ of opioid-naïve Canadians older than 65 years who were prescribed an opioid for a short-stay surgery were identified to be users 1 year after their surgery. A multitude of studies (both multicentre and registry-based) also identify the clear overprescription of opioids following surgical intervention. An audit of more than 7600 patients in the National Surgical 
Quality Improvement Program (NSQIP) reported a median of 375 OME prescribed (equivalent to over 70 tramadol tablets). ${ }^{7}$ This risk increased with advanced age, female sex, non-cancer diagnoses and obesity. It is also clear that prescribing habits vary widely among surgeons and cannot be explained by either patient or disease factors. To this end, overall prescribing patterns across Canada have shown an equally concerning variance. This reality is echoed by many patients, as only $28 \%$ of postsurgical patients use the entirety of their prescribed opioids (and only 9\% formally dispose of their unused narcotics). It is also clear that prescription size (i.e., pill count) is directly associated with opioid consumption, and therefore abuse, after surgery. ${ }^{8}$

It is interesting to contemplate the propagation and precise role of chronic pain services across Canada as well. Although these programs are typical for large Canadian cities, their patient inclusion, exclusion and program discharge criteria vary dramatically from site to site. For example, in some programs a mandatory discharge occurs at 1-year postenrolment. In others, discharge is much sooner. Regardless of funding, these programs can be challenging to access, care for a limited number of patients, and are often perceived (by both the patient and the referring physician) to be less than effective for our most complex patients. In 2016, the CDC stated that there was no solid evidence that opioids were an effective treatment for chronic pain beyond the 6-week mark. ${ }^{9,10}$ This is strong food for thought not only as surgeons consider their own patient populations, but also the reality that up to $20 \%$ of the decline in the American labour force participation between 1999 and 2015 may be linked directly to chronic opioid use.

To address the opiod crisis, the Canadian government has constructed a formal interventional approach that mandates 4 key elements: prevention, treatment, harm reduction and enforcement. While this initiative is deeply commendable and worth contemplation, we also need to do more as physicians and surgeons. The first step in this endeavour is recognizing that prescription opioids play a major role in subsequent opioid misuse and that the risk of persistent opioid abuse following surgery is real.

We must also follow surgical leaders, such as Ken Leslie, Neil Parry, Daryl Gray and Kelly Vogt at the London Health Sciences Centre, who have created, implemented and studied a standardized, multimodal, opioidsparing analgesic pathway to control postoperative pain while reducing postoperative opioid prescriptions among outpatients undergoing general surgical procedures (i.e., laparoscopic cholecystectomy, hernia repair, breast surgery, anorectal surgery). ${ }^{11}$ By triggering a formal program that included preoperative patient education, knowledge dissemination among surgeons, alternative intraoperative pain management (anti-inflammatories, antiemetics, steroids), and strict postoperative analgesia controls (opi- oid prescription for only rescue scenarios that expire after 7 days), they reduced their narcotic prescription rate by $90 \%$ (only $10 \%$ of patients filled the opioid prescription for additional pain control). When extrapolated to Ontario as a whole, the potential amount of narcotic reduction would be impressive (45000 hernia and cholecystectomy procedures per year in Ontario would lead to 1 million narcotic tablets diverted from unintended usage).

Opioid misuse after surgery is clearly a current public health crisis. By using multimodality therapies, reducing our volume of opioid prescriptions (dose, duration and pill count), and educating patients in the preoperative setting, it is clear that surgeons have a central role and responsibility in decreasing patient risk. Now is the time to contemplate each of our own prescribing habits.

\section{Chad G. Ball, MD}

Coeditor, Canadian fournal of Surgery

Competing interests: None declared.

DOI: $10.1503 /$ cjs.011419

\section{References}

1. Government of Canada. Opioids. Available: https://www.canada.ca/ en/health-canada/services/substance-use/problematic-prescription -drug-use/opioids.html (accessed 2019 June 4).

2. Alberta Health. Alberta opiod response surveillance report, 2018 Q4. Alberta Health; March 2019.

3. Gomes T, Mamdani MM, Paterson JM, et al. Trends in high-dose opiod prescribing in Canada. Can Fam Physician 2014;60:826-32.

4. Cleary JF, Maurer MA. Pain and Policy Studies Group: two decades of working to address regulatory barriers to improve opioid availability and accessibility around the world. 7 Pain Symptom Manage 2018;55:S121-34.

5. Compton WM, Jones CM, Baldwin GT. Relationship between nonmedical prescription-opioid use and heroin use. $N$ Engl 7 Med 2016;374:154-63.

6. Brummett CM, Waljee JF, Goesling J, et al. New persistent opioid use after minor and major surgical procedures in US adults. $7 A M A$ Surg 2017;152:e170504.

7. Thiels CA, Anderson SS, Ubi DS, et al. Wide variation and overprescription of opioids after elective surgery. Ann Surg 2017;266:564-73.

8. Hill MV, McMahon BS, Stucke RS, et al. Wide variation and excessive dosage of opioid prescriptions for common general surgical procedures. Ann Surg 2017;265:709-14.

9. Feinberg AE, Chesney TR, Srikandarajah S, et al. Opioid use after discharge in postoperative patients: a systematic review. Ann Surg 2018;267:1056-62.

10. Sun EC, Darnall BD, Baker LC, et al. Incidence of and risk factors for chronic opioid use among opioid-naïve patients in the postoperative period. FAMA Int Med 2016;176:1286-93.

11. Hartford LB, Van Koughnett AM, Murphy PB, et al. Standardization of outpatient procedure (STOP) narcotics: a prospective noninferiority study to reduce opioid use in outpatient general surgical procedures. 7 Am Coll Surg 2019;228:81-8. 